\title{
Conclusion: The Many Technical, Market, Economic, and Practical Aspects of the World of Small Satellites
}

\author{
Joseph N. Pelton
}

\section{Contents}

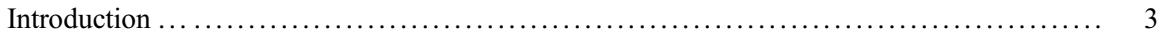

Defining the Various Types of Small Satellites .................................. 5

Small Satellite Technology and Systems ...................................... 6

Design, Manufacturing, Testing, and Resiliency of Small Satellites .................... 8

Launch and Deployment ................................................. 9

Operations and Sparing Philosophy and Space Safety Consideration $\ldots \ldots \ldots \ldots \ldots \ldots \ldots \ldots . .11$

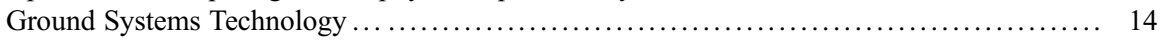

Business, Markets, Financing, Risk Minimization, and Legal Challenges ............... 15

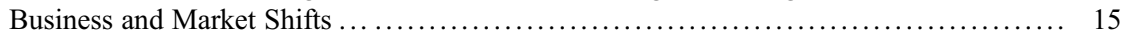

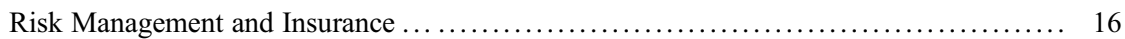

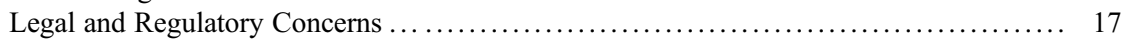

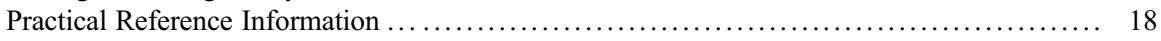

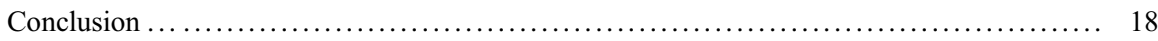

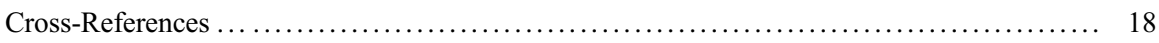

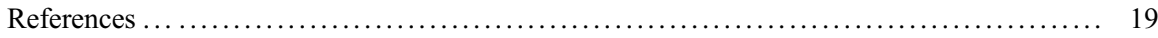

\section{Abstract}

The most significant change in the world of space applications in the past decade is what might be called the small satellite revolution. This small satellite revolution is closely aligned with what is also now known as "Space 2.0" or "NewSpace." Probing discussions of this small satellite revolution and efforts to identify the prime factors that gave rise to this profound change in the space industry produce a number of diverse but convincing answers to what has produced this small satellite revolution. The drivers of this change, largely within the past decade, include (i) new types of flat panel user antennas that can electronically track low Earth orbit satellites in mega-constellations; (ii) new

\footnotetext{
J. N. Pelton (殴

International Space University and Executive Board, International Association for the Advancement of Space Safety, Arlington, VA, USA

e-mail: joepelton@verizon.net
} 
more efficient ways to design and manufacture small satellites at much lower cost and sometimes with the use of off-the-shelf technology; (iii) new lower-cost launchers that can deploy small satellites at much lower cost; (iv) advances in microelectronics, smaller but higher performance sensors, improved component design and manufacture, etc.; (v) a rapid evolution of space services markets through the creation of new types of commercial satellite applications as well as expanded attempts to provide expanded space services to unserved markets in developing economies - especially in the case of broadband Internet streaming and remote sensing; (vi) entrepreneurial business innovation in the space field, often driven by out-of-the-box thinking from the world of computer services and social media; and (vii) new ways of financing small satellite startup ventures based on mechanism such as "Kickstarter," crowdsourcing, rounds of venture capital funding, and crossover investment in satellite applications from new industries such as from the world of computer and information services, investment banking, and other revenue sources.

This Handbook of Small Satellite has sought to examine in some depth all of the sources of change that has produced the small satellite revolution. It has examined the technical, operational, financial, business, economic, regulatory, launcher, and institutional aspects of this important new world of space applications. Innovation is everywhere. There are actually contributions not only on the technical and operational side but from every other side of the business as well. Thus change has come from the small satellite business (i.e., new entries and startups that operate on completely different business models and timetables). There are key changes, from the world of manufacturing and design (i.e., additive manufacturing and use of off-the-shelf components). The small satellite business models have helped reinvent the world of space business and finance that is quite different from the approach taken by giant aerospace companies born of the so-called world of the military-industrial complex. Thus new business practices from the world of small satellites reflect many new patterns of thinking (i.e., new sources of financing and "clean enough rooms"). This new type of entrepreneurial thinking has led to many new ideas such as about sparing philosophies and rapid prototyping and new generations of satellite design in months rather than years. Small satellites have, in short, shaken up thinking throughout the space industry, and change has percolated almost everywhere one might imagine - and then some.

This final chapter seeks to sum up the many areas of change and innovation that have been born of the new world of small satellites. Thus this concluding chapter is divided into discussing important new aspects of the world of small satellites that have permeated the entire space industry. These various sections that are drawn from the component parts of the book include defining the various types of small satellites; satellite technology; design and manufacturing; launch and deployment; operations and sparing philosophy; ground systems technology; business, financing, risk-minimization, and insurance; and regulatory, safety, and institutional issues. 
This chapter concludes with some notes about the practical aspect that are available to readers of the handbook. This includes some guidance with regard to what detailed information is available concerning actual small satellite systems that have been deployed and projects to test new technology or to address space debris concerns. This includes some background with regard to information provided in Section 13 related to small satellite businesses, launch vehicle providers, small satellite networks deployed or planned to be deployed, registration processes related to small satellite systems, the UN sustainability guidelines, and how small satellite systems might relate to the meeting of these goals.

Finally, this conclusion underscores how dynamic both the small satellite market and the launch vehicle systems newly designed to support the launch of small satellites is at this early stage of development. Bankruptcies, mergers, and other realigns are already happening and will continue to occur. The COVID-19 pandemic has already served to accelerate the trend with small satellite company One Web becoming one of the first to fall.

\section{Keywords}

Additive manufacture $\cdot$ Broadband networking $\cdot$ Chip satellites $\cdot$

Crowdsourcing $\cdot$ Cube satellites $\cdot$ Deorbit guidelines · Design and manufacture · Electronic tracking of satellites - Entrepreneurial businesses - Femto satellites · Flat panel antennas - Inter-Agency Space Debris Coordination Committee (IADC) - International Telecommunication Union (ITU) · Launch vehicles · Low Earth orbit (LEO) · Liability · Mega-constellations · Microsatellites ·

Minisatellites ' Nanosatellites · Phased array antennas · Pico satellites - Off-theshelf components $\cdot$ Rapid prototyping - Regulatory provisions - Small satellites · Space applications · Space markets $\cdot$ Sparing philosophy $\cdot$ UN Committee on the Peaceful Uses of Outer Space (UN COPUOS) · Venture capital

\section{Introduction}

The very first satellites that were launched into orbit such as Sputnik 1, Explorer 1 , Score, etc. were, in fact, small satellites. The rapid growth of demand for expanded satellite services for telecommunications, remote sensing, meteorology, and especially the concept of human space travel and the development of larger launch vehicles quickly led to more and more massive satellites to be launched. The pattern of larger and more capable satellites launched by larger and more powerful satellites continued for at least four decades. The advantages of communications satellites operating from Clarke orbit so that ground stations did not have to track the geostationary spacecraft that seemed to hover overhead tended to sustain that pattern of more powerful satellites with larger, high gain antennas for many years.

A combination of factors that evolved in recent years has tended to favor a new focus on smaller satellites for testing new technologies and for experimentation (by students and scientists). There has now been a rapid growth in small satellites for 
many different reasons. This has led in particular to the building of lower-cost small satellites to be deployed low Earth orbit for a variety of new commercial applications. Microelectronics, smaller but higher quality sensors, lower-cost components, new manufacturing techniques and processes, lower-cost launch vehicles, and new phased array, and flat panel antennas that can be used for satellites and also for ground user systems, all support the feasibility of small satellites for commercial networks such as broadband networking via so-called mega-constellations. Now many thousands of small satellites are proposed for launch in these small satellite constellations for broadband networking, remote sensing, and other entirely new services such as RF geolocation, data relay, automatic identification systems, and more.

Currently, there are less than 2000 operational satellites in Earth orbit. If all of the proposed networks of small satellites were to be launched in coming years, the number of operational satellites would increase by a factor of over ten times. On the one hand, these seem to offer new opportunity for a variety of new lower-cost satellite services to be offered on a global basis and reduce the cost of services for communications, networking, remote sensing, and more. On the other hand, there are serious concerns about the rapid expansion of small satellite constellations in Earth orbit of potential space debris and an increased rate of orbital collisions that could greatly accelerate the creation of new debris and threaten the opportunity of access to space in future years.

The prospect of new lower-cost space services, on the one hand, that is offset by serious concerns that large-scale mega-constellations leading to additional space collisions and rapid debris buildup is creating concern about what these new deployment of small satellites portend for the future.

This Handbook of Small Satellites has sought to provide a comprehensive set of information about all aspects of these new small satellite systems and their threats and opportunities to future space services. It also notes that volatility in the markets will continue for the decade ahead. Technological, market, and regulatory changes plus competitive suits will create churn, consolidation, and bankruptcy.

The COVID-19 pandemic that occurred in 2020 has caused health and economic consequences of staggering worldwide consequences that will accelerate the instability in the small satellite markets. This horrific pandemic will impact the small satellite and launch industry in the months ahead. Already LeoSat and OneWeb small satellite constellations have declared bankruptcies and others will follow. There will be more failures of small satellite constellations, launcher companies, and other associated space services companies. Despite these economic failures, the innovative new small satellite technologies, the new more efficient launch systems, the new ground systems, and the many other innovations discussed in this handbook remain valid and very useful sources of new enterprise in this field. In the late 1990s, the original small satellite constellations saw major economic collapses. The economic failures constituted by Iridium, Globalstar, ICO, and Orbcomm made a huge impact on satellite development and the ready access to capital financing for some time. Yet recovery was achieved in the years that followed. The same seems likely to 
occur in this instance as well. Despite these setbacks, the information in this handbook remains useful and quite relevant.

It is, nevertheless, best to consult current websites to chart the latest status of the companies seeking to compete in these markets.

\section{Defining the Various Types of Small Satellites}

One of the least clear terms in the field of space systems is that of a small satellite. There are truly small satellites in range of nanosats and below. These diminutive satellites are often used for the proof of concept or testing of new satellite technologies and systems or student or scientific experimentation. These quite small satellites include femtosats in the range of $10-100 \mathrm{~g}$, picosatellites from $100 \mathrm{~g}$ to $1 \mathrm{~kg}$, and nanosat that range in mass from 1 to $10 \mathrm{~kg}$. Nanosats more or less include 1-6 unit cubesats. Other terms that are in common use are "chipsats" that are usually femtosats and "pocket satellites" that are one-eighth the size of a cubesat and are typically picosats. Increasingly, we are also seeing the deployment of commercial constellations that operate with satellites that are often 3 unit cubesatellites such as those deployed by Planet or Spire. In this handbook, there have been a number of articles that address the design, operation, and usages related to cubesats/nanosats and below and many other articles that address the larger microsats $(10-100 \mathrm{~kg})$ and minisats. Minisats are sometimes referred to as $100-500 \mathrm{~kg}$ and sometimes referred to as $100-1000 \mathrm{~kg}$. Most of the large-scale or mega-constellations for broadband networking, mobile communications, or radar sensing are in these larger classes of "smallsats." The satellites for optical remote sensing, data relay, machine-tomachine (M2M), Internet of Things (IoT), or automatic identification systems (AIS) can range from cubesats/nanosats to microsats. These are, however, only general approximations.

The point is that "smallsat" is truly a very broad term of art. One must know a number of details before one can understand what is actually being referred to as a "smallsat." Key characteristics include such aspects as mass, physical dimensions in stowed and deployed conditions, power, operational radio frequency spectrum utilized, function or service provided, orbital characteristics, and stabilization, orientation, and thruster capabilities. In the increasingly complicated world of small satellites, there are other aspects to consider.

Some "smallsats" are not free flyers. They can actually be "hosted payloads" that can be launched on board a larger to medium-sized satellite. Alternatively, a very small payload such as the Aireon constellation, for ASD-B aviation navigation services, can be deployed on board another small satellite constellation. In this particular case, this small payload was hosted on the 66 satellites of the Iridium NEXT mobile satellite network.

Yet another alternative in terms of small satellite-type capability is not launching of a small satellite at all. Thus, one might deploy high-altitude platforms (HAPS) or stratospheric systems that operate in proto-space or subspace. Such platforms can provide telecommunications, remote sensing, or other services from high-altitude 
Fig. 1 Both sides of chipsat or femtosat that range in size of $10-100$ g. (Graphic courtesy of the global Internet commons)

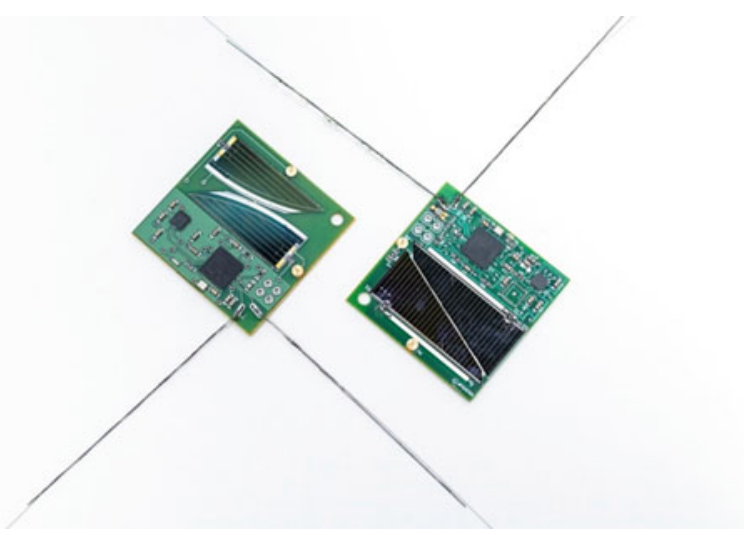

location in order to provide services that are quite similar in concept to a satellite operating at low altitudes. Thus relevant information and some analysis have also been presented in this handbook on both hosted payload systems and high-altitude platforms.

The first chapters in this handbook have sought to provide useful definitions and typical types of functions for various kinds of small satellites that range from femtosats to minisats. This is a gigantic range to contemplate. Femtosats, as represented by a chipsat, begin on the lowest mass of $10 \mathrm{~g}$ (Fig. 1). The highest end of the "smallsat" range is the minisat. These satellites can be as massive as $500 \mathrm{~kg}$ to even $1000 \mathrm{~kg}$ (Fig. 2).

This great dynamic range in size represents five orders of magnitude of scale. This is a differential divide represented by the relationship between 1 and 100,000. This is more or less equivalent to the size difference between a mouse and a hippopotamus.

\section{Small Satellite Technology and Systems}

The amazing development of small satellite technology and systems has enabled smaller and smaller satellites to be developed to carry out an ever-expanding range of services in the fields of telecommunications, networking, remote sensing, data relay and machine-to-machine (M2M) communications, RF geolocation, and scientific experimentation. Some of the innovations are a direct application of new commercial devices and components developed in microelectronics; phased array antenna design; optical processors; new types of optical, near infrared, and infrared sensors; and other new electronic and optoelectronic inventions. Other systems and subsystems are specifically developed by small satellite designers, integrators, and manufacturers seeking to provide more cost-efficient and compact components for small satellite systems. Advances have come from every possible angle and perspective. 


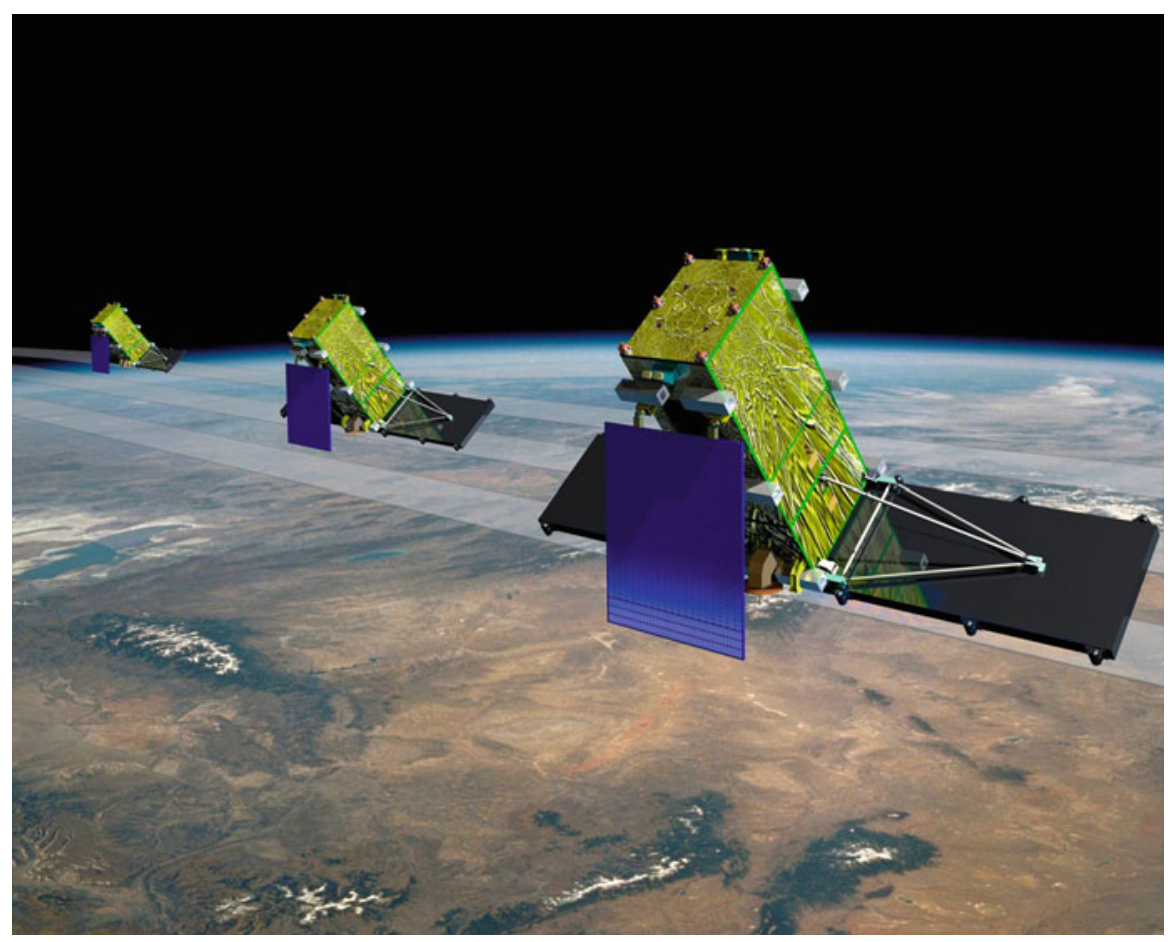

Fig. 2 New Trio of Canadian Radarsats - each minisat with a mass of about $550 \mathrm{~kg}$. (Graphic courtesy of the Canadian Space Agency)

There are improvements everywhere. There are enhanced miniaturization and lower-cost components and sensors. Devices with higher spatial, temporal, or radiometric resolution enhanced digital processing or encoding techniques or simply better or higher throughput production systems for these devices. There are improved miniaturized radio devices and sensors; improved navigation, stabilization, orientation, and pointing systems; better digital communications processing and encoding units; shrunk application-specific integrated circuits; better stabilization systems and torque rods; and improved and lower-cost batteries, social cells, and, coming soon, quantum dot solar power systems.

Part 3 of this handbook details the many technical innovations that are being developed in every aspect and subsystem associated with small satellites. These many innovations are linked to the development of smaller, more efficient, higher throughput, higher resolution, and in some cases lower-cost smallsat components. Innovations at every level of design and subsystem technical improvement are needed to create lower-cost and higher performance small satellite units.

Application-specific integrated circuits (ASICs), digital processors (electronic and optical), advanced encoding systems and software (e.g., turbo coding), advanced sensor design, improved smallsat components of all types, and small satellite kits have facilitated the ability of more and more students, experimenters, and even small 
satellite operators to build small satellite systems more quickly, at lower cost, with greater reliability and with fewer delays. In order to explore specific technical innovations related to the various subsystems involved with the engineering and optimization of small satellites, refer to the chapters in Part 3 of this handbook.

Although improvements in the technical design of many components included in small satellites has led to significant advances, it should also be remembered that satellites, both big and small, are essentially software-defined digital processors in the sky. This means that many of the advances in performance, cost-efficiency, and capability derive from improved software and processing and coding efficiencies. In short, advances are combination of both better hardware and software upgrades as well.

\section{Design, Manufacturing, Testing, and Resiliency of Small Satellites}

A great deal of the progress that has been made in developing higher performance, higher efficiency, and more cost-effective small satellites has been due to technical improvement of the components involved in small satellites, or enhanced software that allows these satellites to achieve faster throughput, spectrum efficiency, or improved preprocessing of remote sensing data. There are other types of improvements related to manufacturing, testing, design processes, and approaches to resiliency that are also key to improvements in small satellite systems. These innovations are more closely related to process and management concepts that technology per se.

Companies such as Planet, Spire, and other constellations that are deploying three-unit cubesat-type satellites employing a different type of approach to manufacturing and testing that employed by commercial operators who typically order a limited number of large GEO type satellites costing many millions of dollars and which take a number of years to design, manufacture, test, and arrange for launch. These small satellite companies take more of an incremental approach.

It is based on constant improvement in the design and manufacturing of its satellites. There is greater reliance on in-orbit testing of a design that is improved almost satellite to satellite and reliability and performance enhanced based on orbital experience. A network that is based on hundreds of satellites with a mean time to failure of perhaps 3-4 years involves a much different approach to design, manufacturing, reliability, and quality testing and resiliency of a so-called megaconstellation. This type of approach allows the possibility to additive manufacture of component parts that can be enhanced with each production run of small satellites.

Particularly in the area of remote sensing, one batch of small satellites might have a slightly better optical sensor, and another might have an improved battery or better efficiency type of solar cell. With a large number of small satellites constantly in production, efforts can be made to improve the reliability of a satellite design based on monitoring the cause of any in-orbit failures. If each small satellite launched into orbit has a net cost of $\$ 100,000$ rather than $\$ 250,000,000$, then the approach to quality testing, design innovation, manufacturing, sparing philosophy, and resiliency changes dramatically. 


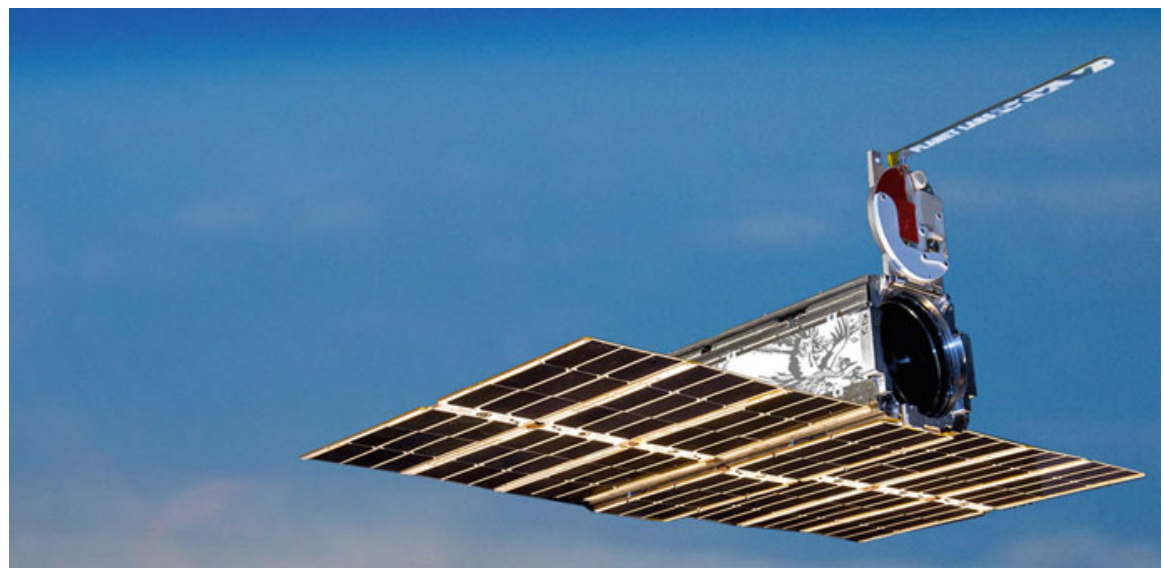

Fig. 3 The Planet LLC Dove 3 U Cube Satellite has now had 18 generations of design innovation and in-orbit testing. (Graphic courtesy of Planet LLC)

Those commercial systems that are deploying hundreds if not thousands of telecommunications or networking minisats for large-scale constellations (i.e., smallsats in the $100-500 \mathrm{~kg}$ class) might require more homogenous designs and more quality testing in their approach to satellite production than that used by Planet or Spire. Nevertheless, their design, manufacture, and testing operations will tend to be more like the production of a television set or aircraft, than a handcrafted electronic product that goes through years of quality testing as was the case with large GEO satellites. In essence, part of the small satellite revolution has come from entrepreneurial insight and even rebelliousness that has concluded the ways of the past are no longer the best way forward. Small satellites such as the Planet Dove, produced in large numbers for LEO constellations, can lead to faster rates of innovation, more in-orbit testing, new modes of production, and even competitive designs (Fig. 3).

\section{Launch and Deployment}

Another part of the "Space 2.0" or the "NewSpace" revolution is not only small satellites but new low-cost commercial launch systems. There are scores of new launch service companies that are offering new types of launchers designed to offer launch services geared to accommodate small satellite missions. Rocket Labs, Vector, and Virgin Orbit's LauncherOne are some of these new rocket systems.

As Virgin Orbit has noted in its promotional materials: "Small satellites are ushering in a new era of space capabilities - connecting us across vast distances, stimulating the global economy, and expanding the limits of human knowledge. This rapidly growing industry requires a launch service that is as agile and affordable as the satellites themselves. But until now, there hasn't been one." The implication is that the LauncherOne rocket system is the solution (Virgin Orbit 2019). The issue of 


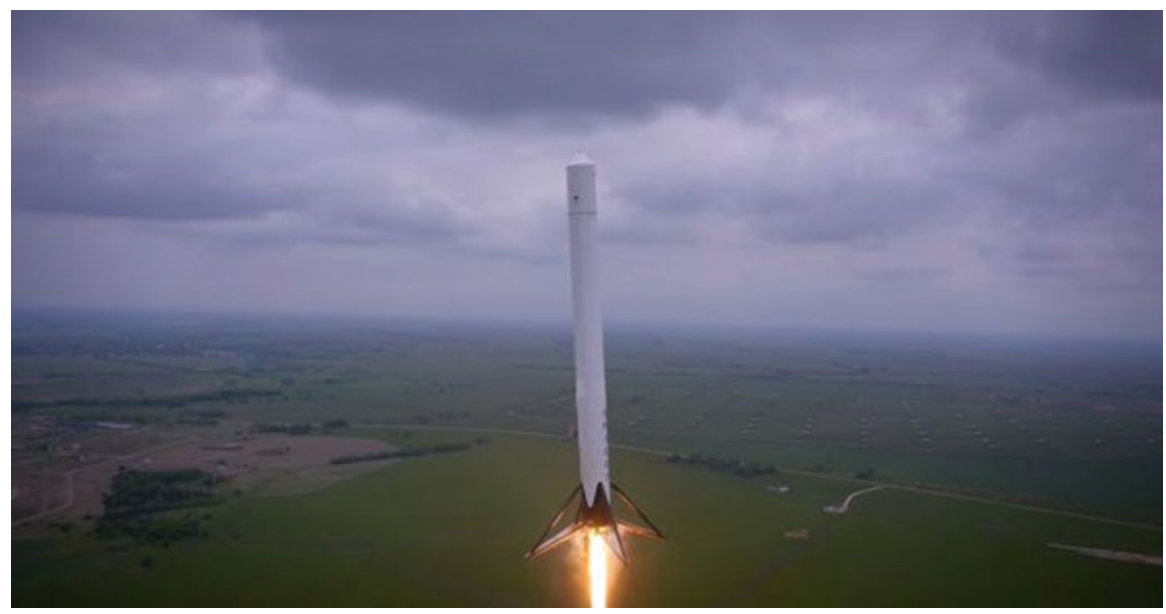

Fig. 4 Test flight of the Falcon 9 Reusable (F9R) launcher that promises new economies for smallsat launches. (Graphic courtesy of SpaceX)

launch arrangements for small satellites is not only that of the convenient availability of launch dates, but it is also cost-effectiveness and reliability of the launcher services. There are indications that larger launch systems might be able to respond effectively to all three aspects of convenience of scheduling, cost competitiveness, and reliability of service.

Conventional launch service providers as characterized by Soyuz/Progress, Ariane, United Launch Services, etc. are seeking to upgrade the launch operations to make them more cost-effective and flexible as to schedule, but the advent of new commercial launch services that employ reusable rockets seems to be particularly well positioned to cater to small satellite launch requirements.

Some analysts have suggested that the biggest innovation in terms of new costeffective launch arrangements is, in fact, coming from reusable launcher systems that are being developed by SpaceX (i.e., Falcon 9R) and Blue Origin (i.e., New Glenn). These launch systems that are designed to recover launcher stages and then reuse perhaps 25 times or more offer the promise of major cost economies for a wide range of launcher missions. Not only are they offering what seem to be very reliable and cost-effective services, but there are new launch schedules now being offered that will launch once a month and reserve a portion of the launch to accommodate small satellite requirements (see F9R launch vehicle in test flight).

And there are now competitive responses from Ariane with its proposed Themis launcher that is now planned to be available by 2023 which is 2 years earlier than the original planned date of 2025 (ArianeGroup 2019). Further Airbus has announced plans for its new Adeline winged booster stage which is under design development (Airbus Enters 2019) (Fig. 4).

What is clear is the cost to launch a cubesat into orbit will continue to come down and that the flexibility to launch based on the schedule of the smallsat clients will 
also likely continue to improve. What is unclear is what will be the ability of reusable vehicles to truly achieve 25 reflights with the same launcher stages and the reliability of these reusable systems. Also unclear is whether the truly small launcher systems such as Vector, LauncherOne, and Rocket Labs will be able to remain competitive against the larger reusable systems now active or planned by SpaceX, Blue Origin, Ariane, Airbus, and perhaps others.

\section{Operations and Sparing Philosophy and Space Safety Consideration}

There are now many student and scientific experiments, test flights of new technology and systems, and one off small satellite missions that will continue to fly into space that are one of a kind missions. If there is a launch failure or the smallsat for some reason does not perform, then the experiment or test flight will simply be tried again. These small satellites typically have no redundancy or backup capability. They often use off-the-shelf components, and there are no planned backup capabilities to keep costs to the minimum. A majority of these systems have an in-orbit lifetime of less than 3 months, and they are often deployed at a low altitude so that they naturally degrade from their orbit and burn up on reentry. The technical and reliability model for these types of experiments and test flight is clear. No sophisticated sparing, operational resilience plan, or active deorbit process is needed. It is possible that there might be some sort of passive device to create atmospheric drag that might be deployed at end of life to facilitate early deorbit.

The small satellites that are deployed as operational constellations, whether commercial, governmental, or defense related, are a different matter. The planning for network reliability, safe deployment, and safe removal from a constellation is a matter of some importance. Indeed since some of these large networks involve capital investments that will exceed in excess of an estimated ten billion dollars (e.g., the SpaceX Starlink and V-band networks) and also involve significant safety risks to long-term sustainability of space, the risk elements are enormous.

There have been new ways to look at the reliability and resilience of satellite constellations. The design and introduction of the mobile satellite networks as represented by the Iridium and Globalstar constellations involved system planning that relied to some extent on deploying orbital spares. These networks had sufficient satellites in orbit that there was thought that holes in the network could be worked around until replacements deployed. This was particularly the case for the Iridium satellite network that had the additional feature of intersatellite links. The use of intersatellite links to work around failed satellites was also envisioned in the case of the proposed MegaLEO constellation known as Teledesic. Furthermore, the second generation of mobile voice communications systems, i.e., Iridium NEXT and Globalstar (OG2), has intersatellite links to minimize ground system investment and the ability to work around in-orbit satellite failure.

The latest planning for operational system reliability for large megaconstellations has focused on using the entire deployed system as the sparing 
Table 1 Possible collision assessment from mega-constellation as conducted by the Aerospace Corporation (Aerospace Corporation)

\begin{tabular}{l|l|l}
\hline $\begin{array}{l}\text { Future } \\
\text { constellation } \\
\text { model }\end{array}$ & $\begin{array}{l}\text { Probability of a collision over 10-year } \\
\text { period during operations }\end{array}$ & $\begin{array}{l}\text { Probability of a collision over } \\
10 \text {-year period during disposal }\end{array}$ \\
\hline FCM 1 & 8 & 15 \\
\hline FCM 2 & 0.5 & 1.25 \\
\hline FCM 3 & 1.1 & 0.6 \\
\hline FCM 4 & 0.33 & 0.55 \\
\hline FCM 5 & 1.25 & 0.80 \\
\hline
\end{tabular}

concept as the size of these networks has increased to hundreds or even thousands of satellites. The sparing philosophy for large small satellite constellations seems adequate to provide for reliability of service for these systems.

The problem of safety and avoidance of potential satellite collisions in the case of very large constellations remains. Studies carried out by the Aerospace Corporation for five of the largest low Earth orbit constellations found that there were significant risks of orbital collisions associated with the initial deployment of these systems as well as significant risk of collision as satellites are removed from service at end of life and deorbited.

This statistical analysis considered the potential risk of a collision occurring over a 10-year period first in terms of the deployment and operational period and second during the removal and deorbit phase for constellations where detailed filing information as to number of satellites, orbital configuration, and density of satellites per orbital configuration. The results of those calculations are shown in Table 1.

This analysis, of course, had to make a number of assumptions in this modeling process, and there is no indication of the level of accuracy that is attributed to the calculations. Nevertheless, even if one assumes that the risks of potential collision have been overestimated by a factor of 2 , there is substantial reason for concern that during operations and disposal operations, the risks of collisions seem to be substantial. This analysis also shows that there is an overconcentration in the current filings of LEO satellite constellations that are to be deployed between 850 and $1000 \mathrm{~km}$ altitudes (Muelhaupt et al. 2019). The above and other assessments of the ever-growing number of large-scale constellations planned for launch in the next decade have given rise to concerns about space debris removal and efforts to minimize orbital collisions.

There have been calls to revise the UN voluntary guidelines for space debris removal. Many of the operators of mega-constellations have voluntarily committed to rapid removal of satellites at the end of life. The problem with rising concerns about the large number of small satellites now planned to be deployed in large constellations is the lack of system limitations. As the threat of possible orbital collisions rises - both during smallsat constellation systems operation and removal lack of regulatory control comes into clear focus. Currently, there are no effective controls that limit the number of systems proposed or approved at the national licensing procedures or ITU system coordination processes that now limit the 
Table 2 The growing number of smallsat constellations for broadband telecommunications. (A compilation of the author)

\begin{tabular}{|c|c|c|c|}
\hline \multicolumn{4}{|c|}{ Proposed small satellite constellations for broadband telecommunications } \\
\hline Country & System name & Number of sats & Radio frequency bands \\
\hline Canada & CANPOL-2 & 72 & $\begin{array}{l}\text { LEO and highly elliptical Earth } \\
\text { orbit in VHF-, UHF-, X-, and } \\
\text { Ka-bands }\end{array}$ \\
\hline Canada & Telesat constellation & $\begin{array}{l}117 \text { satellites plus } \\
\text { spares }\end{array}$ & LEO in Ka-band \\
\hline Canada & COMSTELLATION & Nearly 800 sats & LEO in Ka-band \\
\hline France & $\begin{array}{l}\text { Thales Group's } \\
\text { MCSat }\end{array}$ & $\begin{array}{l}\text { Between } 800 \text { and } \\
4000\end{array}$ & $\begin{array}{l}\text { LEO, MEO, and highly elliptical } \\
\text { Earth orbit in } \mathrm{Ku} \text { - and Ka-bands }\end{array}$ \\
\hline Liechtenstein & 3ECOM-1 & 264 & $\mathrm{Ku}-$ and Ka-bands \\
\hline Norway & ASK-1 & 10 & $\begin{array}{l}\text { Highly elliptical Earth orbit in X-, } \\
\mathrm{Ku}-\text {, and Ka-bands }\end{array}$ \\
\hline UK & L5 (OneWeb) & $\begin{array}{l}650-750 \text { initially } \\
\text { but in time } 1200- \\
4000\end{array}$ & $\mathrm{Ku}-$ and Ka-bands \\
\hline USA & Boeing & $1396-2956$ & V-band in $1200 \mathrm{~km}$ orbit \\
\hline USA & SpaceX & $4500+$ & $\mathrm{Ku}-\mathrm{Ka}$ band \\
\hline USA & SpaceX & 7500 plus & V-band \\
\hline USA & LeoSat & Initially about 80 & Ka-band \\
\hline USA & Athena-Facebook & & Ka-band \\
\hline USA & Karousel MEO & 20 MEO satellites & Ka-band \\
\hline USA & Kuiper-Amazon & 3236 & Ka-band in three orbital tiers \\
\hline USA & O3b mPower MEO & 24 & Ka-band \\
\hline
\end{tabular}

number of satellites or constellations. Table 2 provides a tally of satellite constellations that are just related to proposed broadband telecommunications and networking services. If one totals the maximum number of satellites that could eventually be deployed according to potential full deployment, the number of smallsats as listed in Table 2 could conceivably tally over 27,000 satellites (Pelton 2019a).

This lengthy table just provides proposed smallsats for broadband communications and networking services. It thus does not include, for instance, many more satellites that are now already deployed or proposed for remote sensing, for narrow band data relay, for RF geolocation, or for mobile communications satellite services. What makes the numbers of proposed satellites listed in Table 2 seem so totally remarkable is that the total number of operational satellites now in service as of the start of 2020 is less than 2000. The launch and deployment of so many satellites, followed by the successful operation and maintenance of such massively sized constellations, and then clean disposal of so many satellites are perhaps the greatest challenge of the decade for the space applications industry. This operational challenge might be matched by the growing regulatory challenge. This would be to find means to control and limit the proliferation of smallsat constellations and to facilitate active disposal of satellites at the end of life to cope with related liability issues. 


\section{Ground Systems Technology}

One of the great miscalculations of those that do not know the space applications industry well is to overemphasize the importance of satellites and launchers and underrate the importance of the ground segment. The ground segment associated with space applications as an industry is three to four times the size of the launch services industry. The key to the future of small satellite industry, particularly in the context of constellations, is much more crucially tied to break through in ground system design and performance than perhaps any other factor.

The first step toward new user antenna systems and technology came with the small satellite constellations in low Earth orbit (LEO) that required user transceivers that could receive signals from horizon to horizon as the Iridium and Globalstar satellite crossed over the sky in about $7 \mathrm{~min}$ or so. These new type satellite receivers were designed to capture signals coming from the sky and relied on the signal processing power and digitally encoded transmission that were provided by means of application-specific integrated circuits (ASICs). These digital transceiver units could not electronically track the satellites, but they still demonstrated that small compact handheld units could nevertheless receive satellite signal for voice communications from LEO orbit.

The next step forward was to develop ground systems that can provide electronic tracking of LEO satellites in a global constellation without the need for active mechanical tracking. This required computer-generated electronic beams that could be generated by new flat panel antenna systems. The trick is to develop this type of electronic tracking transceivers that would be reliable enough to switch from beam to beam at the rate of about once a minute and from satellite to satellite about every $7 \mathrm{~min}$ and also be produced at low cost. Today, there are over 20 companies developing flat panel antennas for users for not only mobile satellite communications but now to support even broadband digital networking services.

As identified in the Northern Sky Research report on Flat Panel Antennas, these companies include ALCAN Systems, Anoki Systems, Ball Aerospace, Boeing, C-Com, GetSat, Gilat Satellite Networks, Kymeta, HiSky, Honeywell, Hughes Network Systems, Isotropic Systems, Omni Wave, Phasor Systems, SatixFy, StarWin, ThinKom, Tianyi Satcom Company, Toshiba, and Viasat. Several other companies deploying large-scale constellations such as SpaceX might become selfproducers. In addition, there are other companies that may serve as integrators for military communications satellite companies (Northern Sky Research 2019).

This is an area that is rapidly developing in terms of technology and in achievable cost reductions. One of the many innovators in this area is Isotropic Systems that claim that their optical processing technology will allow one of the most costeffective designs for low-cost user flat panel satellite antennas (Fig. 5). 
Fig. 5 Isotropic Systems flat panel satellite antenna. (Graphics courtesy of Isotropic Systems)

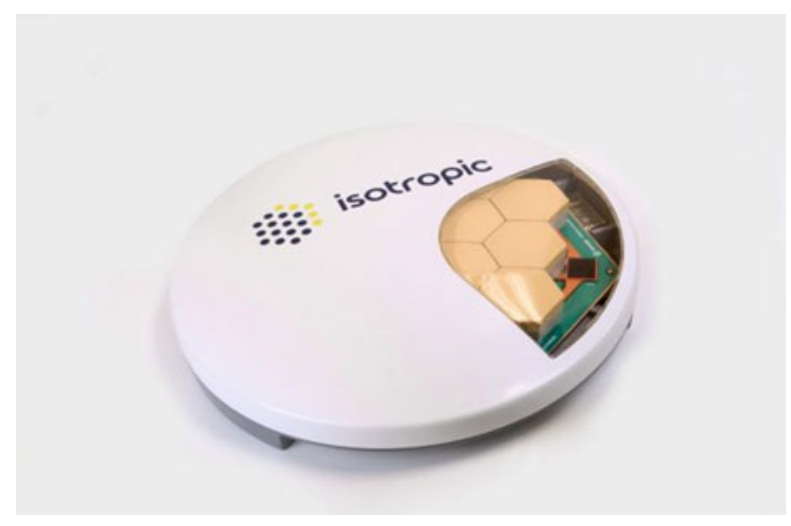

\section{Business, Markets, Financing, Risk Minimization, and Legal Challenges}

Few industries around the world have shown more dramatic changes than that of the small satellite industry. This is not only change in their technologies but also in terms of new business innovation, new business industries, financial change in terms of capital investment, market focus, and even new approaches to risk management and institutional arrangements. Almost anywhere one looks in the small satellite industry, change is afoot.

\section{Business and Market Shifts}

At the level of business innovation, there are scores of new entities identified in Part 13 of this handbook involved in some aspect of the small satellite industry. There are a large number of companies who are involved in designing and building small satellites, in deploying and operating small satellite constellations, in providing launch services for small satellites of all types and sizes, and in manufacturing ground antennas for users and network connectivity. This has created a somewhat chaotic market, and result may see mergers, acquisitions, spinoffs, and even bankruptcies. Companies that were predominant in some space services for remote sensing, telecommunications, broadcast satellite services, or construction of satellites or Earth stations for some decades may see their roles shift, and new innovators take commanding new market leads. The rate of change and the market shifts are still in a state of flux, and predictions of winners and losers are still unclear in this era of rapid market trajectories - some upward and others down.

While this handbook has been in production, the Vector small satellite launcher company, that offered flexible and quick launching opportunities for small satellites, has been forced to declare bankruptcy. LeoSat that was seeking to deploy a 
high-performance, corporate-based broadband service using a small satellite constellation has also declared bankruptcy. Intelsat and OneWeb were planning to merge under a financing plan offered via the Japanese banking giant SoftBank, and now Intelsat has filed a very substantial suit against both OneWeb and SoftBank. Audacy that is seeking to provide data relay services via a new constellation has recently pursued talks with Iceye about possible strategic partnerships to achieve support for its high capital costs to complete its network.

Companies that are pursuing total new markets and services have a great challenge of deploying new products or services and establishing a successful business and market as essentially totally new startups. Thus totally new offerings such as the HAPS Stratobus by Thales Alenia and the RF Geolocation offerings by Hawkeye360 have to balance startup costs versus establishing totally new streams. The bottom line is that many of these small satellite ventures and new launch vehicle ventures could fail. On top of this, the most profitable revenue stream for satellites historically has been broadband broadcast of video services that are now being challenged by over-the-top Internet streaming. One of the large market questions is whether LEO small satellite constellations can find a successful way to offer both $5 \mathrm{G}$ broadband and video streaming services which are the largest potential markets. These are questions that are still unanswered. The bottom line is that small satellite markets are still quite unsettled and market volatility is a part of the small satellite domain. The fact that Silicon Valley giants such as Google, Facebook, and other Internet backers are a part of the story leads to confidence that many of these ventures can succeed, although some may fail.

\section{Finance and Capitalization}

One of the most significant changes is that there are new sources of business innovation and new types of businesses providing innovative sources of capital financing that range from crowdsourcing, Kickstarter, and a number of industrial heavyweights. These include the computer services and networking industries, largely coming from Silicon Valley such as Google, Facebook, Amazon, etc. There are yet others that are heavily involved such as SpaceX and new launch services companies, as well as international banks such as SoftBank of Japan, and others such as Liberty Media, venture capital firms, and others from the Space 2.0 or "NewSpace" industries (Pelton 2019b).

\section{Risk Management and Insurance}

The whole new set of economics associated with the design, manufacture, and operation of small satellites coupled with reduced launch costs and entrepreneurial talent and sense of reinvention has not only reduced the barriers to market entry, but it has created a whole new concept about approaches to sparing philosophy, approach to manufacture and deployment, and strategy related to insurance coverage. The traditional approach associated with high-throughput GEO satellites with very strong cost-effective performance has challenged conventional GEO satellite 
design from one perspective and has moved toward economies of scale achieved with only a few very high-cost satellites. On the other hand, the new small satellite constellations have initiated a new form of economy of scale by the launch of hundreds if not thousands of small satellites. This approach creates a whole different business model. The business concepts can all be different: (i) system design; (ii) the approach to design and procurement of satellites and launch services; (iii) the sparing, the insurance, and risk minimization philosophy; and (iv) design and implementation of the ground system architecture. These issues and more are now all potentially different. New models are being created and new approaches embraced.

\section{Legal and Regulatory Concerns}

The enormous change created by small satellite technology, constellation design, new ground systems, new businesses, and entrepreneurial innovation has created new businesses and new approaches to space application services and innovative new markets. But there are also new and major concerns about the national and international space regulatory environment. There are key concerns about liabilities, space debris, and sustainable space operations within this new environment. The issues and regulatory concerns cut in different directions. On the one hand, there are concerns that the advantages that small satellites can offer to student experimentation, space programs for developing countries, and use of space technology to meet the sustainable development goals of the UN all argue for limits on overregulation of small satellites. On the other hand, the prospect of the number of operational satellites increases by an order of magnitude in the next decade, and the amount of orbital debris in orbit swelling out of control and making the so-called Kessler syndrome a reality could have catastrophic impact.

There is today a lack of new international regulatory authority to cope with the many changes that have occurred since the five international agreements negotiated and agreed within the United Nations Committee on the Peaceful Uses of Outer Space and the General Assembly in the late 1960s and 1970s. The proliferation of small satellites, LEO and MEO constellations, and space debris poses regulatory challenges. The analysis in this handbook suggests that new international agreements to cope with these problems will not likely be achieved in the near term. Thus national procedures to address orbital debris, space debris removal, space situational awareness, and space traffic management will likely need to lead the way forward in the nearer term. These legal and regulatory concerns will play a key role in how the new space industries and the small satellite industries evolve in the coming decade and perhaps the decade to follow. 


\section{Practical Reference Information}

The field of small satellites is evolving rapidly, the dimensions of the smallsat world seem to be expanding every day, and the complexity will only continue to grow. This handbook has sought to cover every aspect of the small satellite revolution in terms of the satellite and ground system technology, the regulatory, business and financial issues, and the practical aspects of various small satellite projects with useful case studies. Part 13 of this handbook provides useful information on small satellite companies, satellite constellations, launch vehicle companies, processes for registration of satellite launches, the UN Sustainable Development Goals, and more.

\section{Conclusion}

The Handbook of Small Satellites is an interdisciplinary, comprehensive, and hopefully useful guide to every aspect of the world of small satellites. If covers the true small satellites used for experimentation and technology demonstration that range from femtosats, picosats, and nanosats on one hand to the other larger and businessoriented systems on the other hand.

It thus covers commercial small satellite constellations that typically range from three-unit cubesatellites up to microsats and minisats. It has sought expertise from around the world from Africa, Asia, Australia, Europe, North America, and South America and scores of space, satellite, and launch vehicle companies around the world. It seeks to cover the technology; the operational, market, and financial challenges; the regulatory shifts; and the fact that the world of small satellites is still volatile and sometimes uncertain. This is always the case with new technology, new markets, and totally new ways of providing services to a global environment that is rapidly shifting.

It has been a collaborative process with careful thought given to every aspect of this complicated new world of small satellites. It is hoped that this reference work proves to be of value to those that consult it as a reference source.

\section{Cross-References}

\section{Analysis of Orbit Debris}

Companies Involved in Design, Manufacture, and Testing of Small Satellites

- Forms for Registration of Small Satellites Consistent with the Registration Conventions

- Global Launch Vehicle Systems for Potential Small Satellite Deployment

- Historical Perspectives on the Evolution of Small Satellites

- Introduction to the Small Satellite Revolution and Its Many Implications

- Partial Listing of Small Satellite Constellations and Related System Infrastructure (See Bryce Report)

- U.N. Sustainable Development Goals for 2030 


\section{References}

Airbus Enters Reusable Rocket Race against SpaceX with Adeline. (2019). https://search.yahoo. com/yhs/search?hspart=trp\&hsimp=yhs-001\&type $=$ Y115_F1_159651_053019\&p=air+bus +adeline+launchpad. Accessed 11 Oct 2019

ArianeGroup Reveals New Launcher Heavily Influenced by Falcon 9. (2019). https://www.reddit. com/r/SpaceXLounge/comments/aw48a6/arianegroup_reveals_new_launcher_heavily/. Last accessed 11 Oct 2019

T. Muelhaupt, M. Sorge, J. Morin, R. Wilson, Space traffic management in the new space age. J. Space Saf. Eng. 6(2), 80-87 (2019)

Northern Sky Research, in Flat panel satellite antennas, 4th edn. (Northern Sky Research, Cambridge, MA, 2019). https://www.nsr.com/research/flat-panel-satellite-antennas-4th-edition/

J.N. Pelton, Lecture materials, small satellite course (University of Cape Town, Cape Town, 2019a)

J.N. Pelton, Space 2.0: Revolutionary advances in the space industry (Springer Nature, Cham, 2019b)

Virgin Orbit. (2019). https://virginorbit.com. Last accessed 10 Oct 2019 